\title{
Estudio de verbos copulativos a partir de corpus de aprendices*
}

\author{
Hui-ChuAn Lu \\ NCKU, TAIWÁN \\ An Chung Cheng \\ Universidad de Toledo, USA
}

Received: 26 July 2013 / Accepted: 12 August 2014

ISSN: $1697-7467$

\begin{abstract}
RESUMEN: El presente trabajo se concentra en el estudio de los verbos copulativos SER/ ESTAR desde la perspectiva de la lengua aprendida por los estudiantes taiwaneses cuya lengua materna es chino. Llegamos a las siguientes conclusiones: (1) La incorrecta omisión decrece durante el proceso de desarrollo. (2) El uso de SER de los estudiantes de niveles más avanzados es más correcto que los iniciales. (3) $S E R$ se aprende más temprano y mejor que ESTAR. (4) El uso correcto de ESTAR en la estructura ESTAR+Adjetivo es relativamente difícil. Los taiwaneses se asemejan a los nativos ingleses como se propuso en los estudios anteriores.
\end{abstract}

Palabras clave: corpus de aprendices, verbo copulativo, omisión, adjetivo

\section{A Learner Corpus-Based Study of Copular Verbs}

\begin{abstract}
This study investigated the acquisition order of the Spanish copular verbs, $S E R / E S T A R$, by students in Taiwan, whose first language is Mandarin Chinese. The findings suggested (1) the incorrect use of copular omission gradually decreased as learners' proficiency levels increased; (2) The usage of SER by more advanced learners is more accurate than that of beginning-level learners; (3) SER is acquired before ESTAR; (4) the structure of $E S T A R$ with adjective is more difficult to be acquired than other uses of copulas. The findings corroborate with previous studies on the acquisition order of the Spanish SER/ESTAR.
\end{abstract}

Keywords: learners' corpus, copular verb, omission, adjective

\section{INTRODUCCIÓN}

$\mathrm{Al}$ adquirir una lengua diferente de la nativa, el aprendiz siempre confronta varias dificultades que intervienen en el proceso de desarrollo, así que unas estructuras de la lengua meta se adquieren más tarde que otras. La situación se hace obvia cuando la lengua adquirida y la nativa son lenguas con aspectos lingüísticos muy diferentes, tal como el sistema de verbos copulativos en español y chino. Además, como los aprendices con distintas experiencias de adquisición pueden variar en los resultados de desarrollo, querríamos examinar si las propuestas de los estudios anteriores pueden generalizarse a los estudiantes cuyas lenguas

*El presente trabajo se basa en el proyecto de investigación apoyado por el National Science Council de Taiwán (NSC96-2411-H-006-028-MY2). 
maternas difieren, aprovechando los poderosos recursos de corpus con el fin de obtener eficazmente y de manera más sistemática conclusiones para facilitar la enseñanza en el futuro.

Esta investigación, mediante la ayuda de un corpus construido y una herramienta auxiliar, se concentra en el estudio de los verbos copulativos SER y ESTAR desde la perspectiva de la lengua aprendida. Basándonos en el sub-corpus del Corpus Escrito de Aprendices Taiwaneses de Español (CEATE), analizaremos los verbos copulativos que usan los alumnos de distintos niveles de aprendizaje, estudiando los datos de sus redacciones, las cuales han compuesto a partir de unas imágenes que se les ofrecen previamente. Aprovechando la herramienta de UAM CorpusTool', los datos se han anotado por "correcciones o errores" y "contextos variables u obligatorios" con respecto a los usos de los verbos copulativos en español.

El presente trabajo se organiza como lo sigue. La segunda sección se dedica a repasar las investigaciones antecedentes al presente estudio. En la sección 3, se presenta la metodología adoptada para realizar el estudio. Señalamos los resultados y el debate en la sección 4. Y concluimos el estudio en la quinta sección.

\section{Estudios previos}

\subsection{Distribución lingüística de verbos copulativos en español, inglés y chino}

A pesar del desacuerdo de la definición exacta de un verbo copulativo, en sentido común, es considerado como un conector entre el sujeto (o un referente) y un predicado, que puede ser una frase sustantiva, un adjetivo, un constituyente usado como un complemento de sujeto o un adverbio. En el caso del español, ambos verbos, SER y ESTAR, pueden vincular un referente a un sustantivo, un adjetivo, un adverbio, una preposición, un gerundio, o un participio pasado. La selección de un verbo copulativo entre SER y ESTAR depende de la estructura sintáctica, la opción léxica, la restricción semántica de un referente o un predicado, y la pragmática de contexto. Es decir, SER y ESTAR difieren sintáctica, semántica y pragmáticamente.

En el idioma español, los verbos copulativos SER y ESTAR, corresponden a "to be" en inglés. En chino, estos verbos copulativos españoles ocupan la posición pre-adjetival y las formas equivalentes en chino pueden ser "SHI, BE...LE/GUO, ZHENG(ZAI), ZHE, ZAI" o nada según los diferentes contextos. Las siguientes oraciones (1a)-(4a) muestran las posiciones donde sólo SER y sólo ESTAR puede usarse. Esto se basa en los usos comunes presentados en los textos estudiantiles. Las oraciones (b) y (c) son las traducciones correspondientes en inglés y chino.

A. SER+SN (Sintagma Nominal)

(1a) Elena es profesora.

(1b) Elena is professor.

(1c) Elena SHI jiaoshou.

B. SER + SPrep (Sintagma Preposicional)

${ }^{1}$ Agradecemos el uso gratis del UAM CorpusTool desarrollado por Mick O'Donnell. 
(2a) Julio es de México.

(2b) Julio is from Mexico.

(2c) Julio SHI cong Mexico lai DE.

C. SER+Participio pasado (Voz Pasiva)

(3a) La casa fue pintada.

(3b) The house was painted.

(3c) na fangzi BE youchi LE/GUO.

D. ESTAR+Gerundio

(4a) Juan está cantando.

(4b) Juan is singing.

(4ci) Juan ZHENGZAI changge.

(4cii) Juan ZHENG changZHEge.

Las oraciones (5)-(10) señalan la estructura sintáctica donde ambos verbos copulativos SER y ESTAR pueden aparecer. Estas dos estructuras son "verbo copulativo+Sintagma Preposicional/Adverbio (locativo)" y "verbo copulativo+Adjetivo", como se señalan en (5)-(6) y (7)-(10) respectivamente.

E. SER/ESTAR+SP/Adverbio (locativo)

i. Localización de evento

(5a) El examen es en aula A.

(5b) The test takes place in classroom A.

(5c) Kaoshi Ø ZAI A jiaoshi.

ii. Las demás localizaciones

(6a) El examen está en aula A.

(6b) The test is at classroom A.

(6c) Shijuan Ø ZAI A jiaoshi.

Hay tres tipos de adjetivos. (i) Los usados exclusivamente con SER: Algunos adjetivos sólo pueden aparecer con $S E R$, por ejemplo, posible 'possible' y asombroso 'astonishing'. (ii) Los usados exclusivamente con ESTAR: hay adjetivos que ocurren exclusivamente con ESTAR tal como lleno 'full', descalzo 'without shoes', y muerto 'dead'. (iii) Los usados con ambos verbos copulativos SER o ESTAR: Existen unos adjetivos que se pueden usar con $S E R$ o ESTAR, pero los sentidos de la interpretación difieren, se nota un contraste en los sentidos de interpretación. Los ejemplos se muestran en (7)-(10).

F. SER/ESTAR+Adjetivo

i. $S E R+$ Adjetivo

(7a) No es posible.

(7b) It is not possible.

(7c) Bu Ø keneng.

ii. ESTAR+Adjetivo

(8a) Está muerto.

(8b) $(\mathrm{S} / \mathrm{he})$ is dead.

(8c) ta $\varnothing$ si LE. 
Con respecto a la tercera clase de adjetivos, los que pueden combinarse con ambos verbos copulativos, Solé y Solé (1977) aseguran que SER se usa con los adjetivos y participios para caracterizar, definir o describir un referente. En contraste, ESTAR se coloca con los mismos adjetivos para describir los estados. Las diferencias se indican en las oraciones (9)-(10).

iii. SER/ESTAR+Adjetivo

- Característica

(9a) Es alegre.

(9b) She is a happy person.

$(9 \mathrm{c})$ ta $\varnothing$ gexingkailang.

- Estado

(10a) Está alegre.

(10b) She is in a happy mood.

(10c) ta $\varnothing$ xinqingyukuai.

En comparación con los verbos copulativos en español e inglés, los verbos copulativos chinos son invisibles en los contextos de las descripciones de apariencia física, evento y estado, como se ven en (11a-c) y (12a-c). Si el verbo copulativo SHI del chino aparece en estos contextos, se interpreta con el sentido de "énfasis o contraste", como se nota en (11d) y (12d).

(11a) María es muy bonita.

(11b) Maria is very pretty.

(11c) María Ø hen piaoliang.

(11d) María SHI hen piaoliang.

'María SER muy bonita'

"Es verdad que María es muy bonita."

(12a) Está enfermo.

(12b) He is sick

(12c) Ta $\varnothing$ bing LE.

(12d) Ta SHI bing LE.

'Él ESTAR enfermo LE'

"Es verdad que él está enfermo."

\subsection{Etapas de desarrollo}

En primer lugar, desde la perspectiva de la lengua aprendida: Si bien se consideran los verbos copulativos como un tema gramatical primario que suele incluirse en un contenido programado para los principiantes de español, esta inclusión no garantiza en absoluto su aprendizaje (cf. Briscoe, 1995; Bruhn de Garavito \& Valenzuela, 2006; Finneman, 1990; Ramírez-Gelpi, 1995; Woolsey, 2006a, 2006b). Lo mismo sucede con un aprendizaje de nivel avanzado o superior, es decir, confrontan los mismos problemas los que poseen elevados conocimientos tanto en lingüística como en literatura española para lograr alcanzar el estatus de un hablante nativo. Por lo tanto, estos verbos siguen siendo un problema complicado para el aprendizaje de la lengua española (Geeslin, 2003a). 
Estudios anteriores, de los años ochenta y noventa, se dedicaron a la cuestión sobre las etapas de desarrollo en la adquisición de SER y ESTAR por aprendices ingleses del español en varios niveles de competencia. Estos estudios (Gunterman, 1992; Ryan y Lafford, 1992; VanPatten, 1985, 1987) muestran que los adultos nativos ingleses pasaron las mismas etapas de desarrollo en el proceso de adquirir los verbos copulativos españoles.

Entre ellos, VanPatten $(1985,1987)$ propone las fases del desarrollo sobre la adquisición de verbos copulativos. SER se adquiere antes de ESTAR y "ESTAR+Progresivo" es adquirido antes de "ESTAR+Locativo" y "SER+adjetivo". Además, el uso de ESTAR con adjetivo se adquiere después de otros contextos.

Dichas fases se resumen como sigue:

Fase 1. No existen verbos copulativos en la lengua adquirida (*Juan alto.)

Fase 2. En la fase principiante de la adquisición, se nota el uso excesivo del verbo copulativo SER, es decir, reemplaza al verbo copulativo ESTAR (*Ella es estudiar.)

Fase 3. La adquisición de ESTAR+gerundio (Ella está estudiando.)

Fase 4. La adquisición de ESTAR+adverbio de lugar (Sus padres están en Colombia.)

Fase 5. La adquisición de ESTAR+adjetivo (Juan está enfermo.)

Durante los últimos años, la mayoría de los estudios de los verbos copulativos fundamenta su investigación en el aprendizaje del verbo ESTAR con los adjetivos predicativos (Geeslin, 2000, 2002a, 2002b, 2003a, 2003b, 2004; Geeslin y Guijarro-Fuentes, 2004, 2005, 2006; Woolsey, 2006a, 2006b). La razón es que para los aprendices cuya lengua materna es el inglés, la estructura "ESTAR+adjetivos" es adquirida en la etapa final del proceso de aprendizaje.

\subsection{L2 y L3}

Entre los factores que afectan la adquisición de una tercera lengua (hablante multilingüe), tratamos a dos relevantes al presente estudio: distancia de la lengua y el nivel de competencia de la lengua. La distancia de la lengua se refiere a la relación y similitud entre las lenguas adquiridas de los hablantes no nativos. Se propone que para hablantes multilingüe el aprendizaje es irresistiblemente influido por la lengua que es la más cercana a la lengua meta, sin importar si es la lengua nativa o no. Además, los aprendices pueden ser afectados por más de una lengua a la vez (Ringborn, 2007). La influencia cros-lingüística ocurre en una etapa de adquisición más temprana, cuando los conocimientos de los aprendices con respecto a la lengua meta son todavía débiles y fragmentados, y la necesidad de llenar el vacío de conocimiento en la lengua meta es más prensada (Odlin, 1989; Ringbom, 1986; Williams and Hammarberg, 1998: 295), sin embargo, la transferencia también ocurre en niveles más avanzados. En las etapas tempranas de adquisición, la transferencia siempre es negativa porque son los resultados de la estrategia general adoptada para llenar el vacío de conocimiento en la lengua meta. Por otro lado, los efectos de transferencia positiva parecen encontrarse en la etapa avanzada de adquisición, cuando los aprendices típicamente se benefician de sus conocimientos de otras lenguas. Ringbom (1987) propone que la transferencia de forma es un tipo de transferencia relativamente superficial que tiene que ver con la L1 o la L2 igualmente, ya que la competencia en la lengua no-nativa no necesita alcanzar un nivel muy alto para que ocurra este tipo de transferencia. En contraste, la transferencia de sentido sólo puede tener lugar en la lengua no-nativa que los aprendices comprenden muy bien. 
Teniendo en cuenta los estudios antecedentes desde diferentes perspectivas, el siguiente estudio está dedicado a analizar los datos y su distribución, para obtener una generalización de la inclinación que se da en los usos de los verbos copulativos en las diferentes etapas de desarrollo de los estudiantes taiwaneses. Al mismo tiempo, querríamos comparar nuestros resultados con los de estudios anteriores para explicar la gramática universal y los parámetros de lenguas específicas.

Para corresponder a los propósitos de estudio, el presente trabajo pretende resolver las siguientes preguntas:

En el desarrollo de la lengua aprendida,

(1) ¿Qué diferencia hay entre el uso español de SER y ESTAR para los estudiantes taiwaneses que estudian español en diferentes niveles?

(2) ¿Tienen el mismo modelo que los aprendices ingleses los aprendices taiwaneses cuya lengua materna es el chino?

\section{Metodología}

Los métodos que adoptamos para realizar nuestra investigación se componen de varias partes, tanto por lo que se refiere a la estructura en general y la de SER/ESTAR+Adjetivo como al uso y la omisión de los dos verbos copulativos, SER y ESTAR.

En el presente trabajo, estudiaremos la lengua aprendida mediante los usos de los verbos copulativos por los alumnos taiwaneses según distintos niveles en su aprendizaje, analizando los datos de las redacciones suscitadas por dibujos ofrecidos previamente y extraídas del corpus CEATE (Corpus Escrito de Aprendices Taiwaneses de Español).

\subsection{Participantes y datos}

Hemos construido el corpus CEATE que comenzó a desarrollarse en el año 2005. Este corpus ha recopilado 2.425 redacciones (aproximadamente 446.694 palabras) procedentes de 15 universidades de Taiwán en los últimos 9 años. La consulta a esta base de datos está disponible al servicio público mediante el sitio web http://www.corpora.flld.ncku.edu. tw. La motivación de extraer datos del sub-corpus CEATE 2008 procede de la necesidad de incluir datos del mismo tema, con la finalidad de investigar la adquisición de los verbos copulativos sirviéndonos de un método más sistemático, en comparación con las redacciones de CEATE 2005-2007 que contienen temas variados y fueron incluidos en nuestro estudio anterior (Cheng, Lu y Giannakouros, 2008). Las composiciones de CEATE 2008 se realizaron en clase, según las ilustraciones ofrecidas (véase Apéndice). Durante la realización, los participantes deben narrar en 45 minutos dos textos utilizando las palabras y las ilustraciones ofrecidas sin utilizar diccionario ni cualquier otro instrumento auxiliar.

Cabe señalar que los estudiantes que contribuyeron con las redacciones no poseen el mismo número de horas dedicadas al estudio del idioma español. El cálculo se basa en la siguiente fórmula: $\alpha$ horas semanales x 16 semanas $\mathrm{x} \beta$ semestres. Los datos analizados en el presente trabajo se extraen del corpus CEATE 2008. Excluimos a los que han aprendido el idioma español por tiempo menor a 100 horas y tratamos de incluir a participantes de dos grupos principales en Taiwán: los del Departamento de Español y los del Departamento 
de Lenguas Extranjeras. Además, considerando la homogeneidad de profesores, materiales didácticos y la mayor cantidad de textos para comparaciones posteriores, incluimos a los participantes provenientes de tres diferentes niveles de la Universidad Providencia. Los datos analizados están compuestos de cuatro niveles I-IV, con crecientes horas de aprendizaje del español y correspondientes a participantes procedentes del Departamento de Lenguas Extranjeras de la Universidad JiaYi y de la Universidad Nacional de Cheng Kung (128 horas), el primer curso del Departamento de Español de la Universidad de la Universidad Providencia (544 horas), el segundo curso de la Providencia (1152 horas) y el cuarto curso de la Universidad de la Providencia (1856 horas), respectivamente. Para evitar la intervención de factores inesperados, tal como la experiencia especial de aprendizaje, no incluimos los que han aprendido el idioma español de cualquier forma antes de entrar en la universidad, ni a los que han vivido en países hispanohablantes. Además, se excluyen los textos con una longitud menor a 100 palabras. En total, analizamos 119 textos, 19.679 palabras, como se observa en el Cuadro 1.

Cuadro 1: Corrección de verbos copulativos

\begin{tabular}{|c|l|l|c|c|c|c|}
\hline Nivel & Universidad & Departamento & $\begin{array}{c}\text { Años de } \\
\text { aprendizaje }\end{array}$ & $\begin{array}{c}\text { Horas de } \\
\text { aprendizaje }\end{array}$ & Participantes & Palabras \\
\hline I & $\begin{array}{l}\text { NCKU2, } \\
\text { JY2 }\end{array}$ & $\begin{array}{l}\text { Lenguas } \\
\text { Extranjeras }\end{array}$ & 2 & 128 & 17 & 2.680 \\
\hline II & PU1 & Español & 1 & 544 & 12 & 1.980 \\
\hline III & PU2 & Español & 2 & 1152 & 61 & 15.019 \\
\hline V & PU4 & Español & 4 & 1856 & 29 & 7.485 \\
\hline Total & & & & 119 & 19.679 \\
\hline
\end{tabular}

\subsection{La anotación}

Después de recopilar las redacciones, las pasamos al ordenador, luego las archivamos en formato de texto con codificación Unicode UTF-8 para guardar algunas letras y puntuaciones especiales del español.

Al guardar y archivar los datos, anotamos los usos de los verbos copulativos utilizados en las redacciones de los estudiantes y los contrastamos con los textos revisados por los hablantes nativos usando el CorpusTool, sistema diseñado por Mick O’Donnell de la Universidad Autónoma de Madrid (UAM), para facilitar el trabajo de anotaciones y análisis posterior. Con respecto a las variables examinadas, asignamos dos tipos de códigos principales: (1) contexto de hablante nativo y (2) corrección o error de estudiantes. Los usos correctos e incorrectos se verifican mediante los contrastes entre los usos de los aprendices y los hablantes nativos que se encargan de revisar los textos de los aprendices. Incluimos 47 rasgos de 
sub-código para justificar si es un contexto obligatorio (sólo SER o sólo ESTAR) o variable (ambos verbos aceptables) para un hablante nativo, para verificar si nuestro estudiante usa los verbos copulativos correcta o incorrectamente, incluyendo la consideración de omisión en diferentes posiciones (omisión obligatoria e inaceptable) y estructuras $(S E R / E S T A R+\mathrm{SN}$, gerundio, participio pasado, locativo, adjetivo...etc.). En total, realizamos 4.663 anotaciones.

\subsection{Cálculo de usos correctos e incorrectos según diferentes variables examinadas}

En el presente estudio, nos centramos en el análisis de los dos verbos copulativos $S E R$ y ESTAR, considerando tanto los usos como las correcciones de las formas seleccionadas por los aprendices. En el cálculo de corrección, adoptamos el patrón: Usos en los Contextos Obligatorios (UCO) Simplificado, para calcular los resultados anotados, el cual se señala como sigue:

UCO Simplificado en los contextos no-variables $=\mathrm{x} / \mathrm{a}$

$\mathrm{x}$ = números de usos correctos de SER/ESTAR en los contextos no-variables

$\mathrm{a}=$ números de usos de $S E R / E S T A R$ requeridos en los contextos no-variables

Tomando como ejemplo los usos del UCO-SER, la corrección se calcula como: los usos correctos de $S E R$ utilizados por los aprendices en los contextos no-variables divididos por los usos del $S E R$ utilizados por los hablantes nativos del idioma español en los contextos no-variables. Con el fin de observar la inclinación de los usos en diferentes etapas de adquisición, computamos las correcciones según diferentes verbos copulativos y estructuras de distintos niveles.

De forma similar, aplicamos la misma fórmula para calcular las omisiones agramaticales. En este caso, vemos que entre más alto es el porcentaje del resultado, más omisión inaceptable ocurre en los contextos.

Omisiones incorrectas en los contextos no-variables $=\mathrm{y} / \mathrm{a}$

$\mathrm{y}=$ números de omisiones incorrectas de SER/ESTAR en los contextos no-variables

$\mathrm{a}=$ números de $S E R / E S T A R$ requisitos y contextos no-variables

\section{Resultados y debate}

Los resultados del cálculo se muestran en el Cuadro 2, incluyendo las tasas de corrección y los porcentajes de omisiones incorrectas con respecto a dos verbos copulativos, SER y ESTAR. 
Cuadro 2: Corrección y omisión de verbos copulativos

\begin{tabular}{|l|c|c|c|c|c|c|}
\hline $\begin{array}{c}\text { Nivel: horas } \\
\text { de aprendizaje }\end{array}$ & $\begin{array}{c}\text { Participantes: } \\
\mathbf{1 1 9}\end{array}$ & SER & ESTAR & SER+J & ESTAR+J & Omisión \\
\hline $\begin{array}{l}\text { I: } 128 \text { horas } \\
\text { (NCKU2, JY2) }\end{array}$ & 17 & $69,62 \%$ & $40.22 \%$ & $69.62 \%$ & $37.47 \%$ & $9.48 \%$ \\
\hline $\begin{array}{l}\text { II: } 544 \text { horas } \\
\text { (PU1) }\end{array}$ & 12 & $89,09 \%$ & $29,40 \%$ & $89.09 \%$ & $27,28 \%$ & $9,14 \%$ \\
\hline $\begin{array}{l}\text { III: } 1152 \text { horas } \\
\text { (PU2) }\end{array}$ & 61 & $92.32 \%$ & $44,60 \%$ & $90.29 \%$ & $36.52 \%$ & $4,27 \%$ \\
\hline $\begin{array}{l}\text { V: } 1856 \text { horas } \\
\text { (PU4) }\end{array}$ & 29 & $96,14 \%$ & $64,21 \%$ & $92.5 \%$ & $58.62 \%$ & $2.87 \%$ \\
\hline
\end{tabular}

\subsection{Corrección de verbos copulativos y omisión de verbos copulativos}

Según el Cuadro 2, los estudiantes taiwaneses que aprenden el español como la segunda lengua extranjera, se nota que los porcentajes de corrección en los casos de SER superan a ESTAR en todos los niveles: $69.62 \%>40.22 \%, 89.09 \%>29.40 \%, 92.32 \%>44.60 \%$, $96.14 \%>64.21 \%$ para niveles I-IV, respectivamente. Además, se observa el desarrollo creciente en los usos correctos de SER del nivel I al IV $(69.62 \%<89.09 \%<92.32 \%<96.14 \%)$ mientras que se comportan un poco diferente en el caso de ESTAR $(40.22 \%>29.40 \%<44.60 \%<64.21 \%)$ en el cual el nivel I supera al nivel II. Las tendencias similares de desarrollo se mantienen en la estructura de $S E R / E S T A R+$ Adjetivo: $69.62 \%<89.09 \%<90.29 \%<92.5 \%$ para $S E R$ y $37.47 \%>27.28 \%<36.52 \%<58.62 \%$ para ESTAR. En comparación con todos los usos SER/ESTAR en conjunto, los participantes se desempeñan más pobremente en el uso de la estructura $S E R /$ ESTAR+Adjetivo. Además, los participantes alcanzan un relativamente bajo porcentaje de corrección (27.28\%-58.62\%) en la estructura ESTAR+Adjetivo. Las demás estructuras tales como $S E R / E S T A R+$ Gerundio o locativo no se incluyen en el análisis, debido a los pocos casos usados por los aprendices. A partir de estas cifras, nos damos cuenta de que como se propuso en los estudios anteriores sobre los estudiantes cuya lengua materna es el inglés: (1) El verbo SER se aprende mejor o antes del verbo ESTAR. (2) El uso de SER entre los estudiantes de niveles más avanzados es más correcto que sus usos de los de niveles más iniciales. (3) El uso correcto de ESTAR en la estructura ESTAR+Adjetivo es relativamente difícil en el proceso de adquisición.

Por último, la última columna del Cuadro 2 indica los porcentajes de las omisiones incorrectas de los verbos copulativos cuando en realidad son necesarios. Observamos una clara tendencia decreciente en la incorrecta omisión de los verbos copulativos desde el nivel inicial hasta el nivel más avanzado $(9.48 \%>9.14 \%>4.27 \%>2.87 \%)$.

\subsection{Influencia de L1 y L2}

Los verbos copulativos españoles en la estructura SER/ESTAR+Adjetivo, se traducen en "to be" en inglés y se ocupa por un verbo copulativo invisible en chino, como se señalan en 
los ejemplos (11c) y (12c). Para verificar la relación correspondiente entre las tres lenguas, analizamos 13 cuentos paralelos de español, inglés y chino ${ }^{2}$, en total 9.163, 9.522 y 13.816 palabras en las tres lenguas respectivamente. Estudiamos los verbos copulativos españoles $S E R$ y ESTAR y sus correspondientes expresiones en inglés y chino. El Cuadro 3 señala los resultados de la distribución de verbos copulativos en diferentes estructuras y los resultados en la estructura de SER/ESTAR+Adjetivo se muestran en el Cuadro 4.

Cuadro 3: Distribución de los verbos copulativos conjuntos en las lenguas correspondientes

\begin{tabular}{|c|c|c|c|c|}
\hline \multicolumn{2}{|c|}{ Español } & \multirow{2}{*}{ Inglés } & \multicolumn{2}{c|}{ Chino } \\
\hline \multirow{2}{*}{ SER } & $130(60,5 \%)$ & \multirow{2}{*}{ TO BE } & SHI & $48(36,9 \%)$ \\
\cline { 4 - 5 } & & & $\varnothing$ & $42(32,3 \%)$ \\
\cline { 4 - 5 } & & & YYY & $40(30,8 \%)$ \\
\hline ESTAR & $85(39,5 \%)$ & \multirow{2}{*}{ TO BE } & SHI & $9(10,6 \%)$ \\
\cline { 4 - 5 } & & & $\varnothing$ & $30(35,3 \%)$ \\
\cline { 4 - 5 } & & & YYY & $46(54,1 \%)$ \\
\hline
\end{tabular}

Cuadro 4: Distribución de verbos copulativos de la estructura SER/ESTAR+Adjetivo en las lenguas correspondiente

\begin{tabular}{|c|c|c|c|c|}
\hline \multicolumn{2}{|c|}{ Español } & Inglés & \multicolumn{2}{c|}{ Chino } \\
\hline SER+Adjetivo & $56(51,4 \%)$ & \multirow{2}{*}{ TO BE } & SHI & $7(12,5 \%)$ \\
\cline { 4 - 5 } & & & $\varnothing$ & $31(55,4 \%)$ \\
\cline { 4 - 5 } & & & YYY & $18(32,1 \%)$ \\
\hline ESTAR+Adjetivo & $53(48,6 \%)$ & \multirow{2}{*}{ TO BE } & SHI & $7(13,2 \%)$ \\
\cline { 3 - 5 } & & & $\varnothing$ & $22(41,5 \%)$ \\
\cline { 4 - 5 } & & & YYY & $24(45,3 \%)$ \\
\hline
\end{tabular}

En primer lugar, según los resultados del Cuadro 3, observamos que el $63,1 \%$ de los verbos $S E R$ y el $89,4 \%$ de los verbos ESTAR en las estructuras incluyendo $S E R / E S T A R+\mathrm{N} /$ Adjetivo/Adverbio/Preposición no se traduce en verbo copulativo SHI en chino: el 32,3\% se omite y el $30,8 \%$ se traduce en otras expresiones en el caso del verbo $S E R$, mientras que el $35,3 \%$ de los casos el verbo se omite y el $54,1 \%$ corresponde a otras expresiones en el caso del verbo ESTAR. Además, si nos centramos sólo en la estructura SER/ESTAR+Adjetivo, una

\footnotetext{
${ }^{2}$ http://en.childrenslibrary.org/, http://itunes.apple.com/hk/app/id440153337?mt=8
} 
similar distinción también se muestra en el Cuadro 4. Observamos que el $87,5 \%$ de SER y el $86,8 \%$ de ESTAR no se traducen en verbo copulativo SHI en chino, el $55,4 \%$ se omite y el $32,1 \%$ se traduce en otras expresiones en el caso del verbo $S E R$, mientras que el $41,5 \%$ se omite y el $45,3 \%$ corresponde a otras expresiones en el caso del verbo ESTAR. Con esta relación y basándonos en el contraste entre los verbos copulativos españoles y los chinos, deducimos que el L1 (chino) de los participantes puede jugar un papel en las etapas iniciales de desarrollo porque la esperada inclinación decreciente de omisión agramatical aparece a lo largo del desarrollo de una nueva lengua aprendida. En general, el verbo copulativo SHI en chino en la estructura $S E R / E S T A R+$ Adjetivo se expresa sólo cuando la oración implica un sentido enfático o contrastivo, como se muestran en los ejemplos (11d) y (12d). Por lo tanto, es lógico que los estudiantes cometan el error de omitir el verbo copulativo por la influencia de posición vacía en su lengua materna y se explica la disminución de la omisión del verbo copulativo desde el nivel principiante a un nivel más avanzado, si se atribuye a la transferencia negativa de L1. Además, también incluimos el factor propuesto por VanPatten (1987), la omisión de $S E R / E S T A R$ en la producción de los aprendices se parece a lo que ocurre en la adquisición de la primera lengua para los niños españoles porque un verbo copulativo conecta dos elementos léxicos y en realidad carga poco sentido.

En segundo lugar, en el Cuadro 3, nos damos cuenta de que los verbos copulativos españoles sin distinguir SER de ESTAR corresponden a "to be", el único verbo copulativo en inglés. La asociación entre L3 (español) y L2 (inglés) de nuestros aprendices, nos motiva a proponer que el verbo $S E R$ es sobregeneralizado en los casos donde el verbo ESTAR debe usarse. La transferencia negativa de L2 parece intervenir y desempeñar un papel en el proceso de desarrollo.

Por último, en el Cuadro 3, también nos percatamos de que el verbo copulativo SER aparece con más frecuencia $(60,5 \%)$ que el otro verbos copulativo, ESTAR (39,5\%). Lo relacionamos con el hecho de que los participantes cometen más errores en la estructura ESTAR+Adjetivo, y proponemos que a lo largo del desarrollo, los verbos copulativos españoles SER y ESTAR compiten por la misma posición antes del adjetivo y entorpece la decisión de selección. Esto se observa en las expresiones paralelas entre tres lenguas diferentes: dos verbos copulativos en español, uno en inglés y ninguno en chino.

\subsection{Implicación pedagógica y limitación}

Con relación a los resultados deducidos, en la etapa temprana o en el nivel inicial del aprendizaje, se enfatiza la presencia obligatoria de los verbos copulativos, contrastando con la omisión de verbos copulativos en la lengua materna de los taiwaneses. En una etapa más tardía, para evitar la sobreutilización de SER en los casos en los que corresponde ESTAR, las diferentes funciones de dos verbos copulativos deben presentarse, sobre todo, en los casos donde ESTAR debe usarse. Por último, el contraste entre los verbos copulativos en la estructura de SER/ESTAR+Adjetivo tiene que asociarse con los sentidos de contexto, acompañándose por ejemplos auténticos para reforzar el aprendizaje.

Con respecto a las limitaciones del presente trabajo, más participantes de diferentes universidades deben incluirse. En vez de tomar en cuenta las horas de aprendizaje del español, hay que adoptar un estándar objetivo para identificar la competencia de los participantes, por ejemplo, la utilización de exámenes tales como: el Placement Test de Wisconsin o el DELE 
(Diploma de Español como Lengua Extranjera). Además de la estructura ESTAR+Adjetivo que se considera difícil de aprender, más casos de otras estructuras tales como ESTAR+Gerundio y ESTAR+Locativo necesitan ser incluidas en la tarea de ilustración para estudiarse con más profundidad. Con estas limitaciones mejoradas, los resultados del futuro trabajo llegarán a una conclusión más generalizada con valor tanto más práctico como teórico.

\section{Conclusión}

Los diferentes fenómenos lingüísticos entre dos lenguas generalmente causan cierto grado de dificultad al adquirir una lengua nueva, sobre todo si se trata de dos lenguas muy distintas morfológica y sintácticamente. Aprovechado los recursos de corpora, que se han popularizado en casi todas las áreas de la lingüística en los últimos años, se investigó el proceso de adquisición de los verbos copulativos para los aprendices taiwaneses de español como L3 cuyas L1 y L2 son chino e inglés respectivamente, para derivar un modelo sistemático como referencia para la enseñanza futura y a la vez, comparar con los estudios anteriores dedicados a los aprendices cuya primera lengua es el inglés. Mediante los análisis y las observaciones de los datos basados en una tarea ilustrada, como lo que se propuso en los estudios anteriores sobre los aprendices cuya lengua materna es el inglés, llegamos a las siguientes conclusiones: (1) La omisión de los verbos copulativos ocurren en los niveles iniciales con más frecuencia que en los avanzados. (2) $S E R$ se aprende más correctamente que ESTAR, o sea, SER se aprende mejor que ESTAR. (3) Para el verbo copulativo SER, los estudiantes de los niveles más avanzados lo usan más correctamente que los iniciales. (4) La adquisición de ESTAR+Adjetivo es relativamente difícil en el proceso de desarrollo. A pesar de las diferentes lenguas maternas de los aprendices entre los estudios anteriores y este trabajo, comparten algunas similitudes en el desarrollo de adquisición, tales como: la omisión del verbo copulativo, la sobregeneralización de $S E R$ y la dificultad de la estructura ESTAR+Adjetivo.

Atribuimos la omisión de los verbos copulativos españoles por parte de los estudiantes taiwaneses a la transferencia negativa de su L1 (la lengua materna, el chino) donde suelen omitirse en la estructura SER/ESTAR+Adjetivo. Además, la sobregeneraización de SER se debe a la transferencia negativa de su L2 (la primera lengua extranjera, el inglés) en la que sólo existe un verbo copulativo. La incorrespondencia de números de verbos copulativos entre la L1 (chino), L2 (inglés) y L3 (español) de los aprendices taiwaneses y las transferencias explican el proceso de desarrollo y provee referencias para un futuro diseño pedagógico. Los recursos de corpus no sólo facilitan el procesamiento de los datos analizados, sino también proveen una nueva visión para reexaminar las propuestas anteriores.

\section{REFERENCIAS BIBLIOGRÁfICAS}

Briscoe, G. G. (1995). The acquisition of ser and estar by non-native speakers of Spanish. (Unpublished Ph.D. dissertation). University of Pennsylvania: Philadelphia.

Bruhn de Garavito, J., and Valenzuela, E. (2006). "The status of Ser and Estar in late and early bilingual L2 Spanish", in C. Klee and T. Face (eds.), Selected proceedings of the 7th 
conference on the acquisition of Spanish and Portuguese as first and second languages. Somerville: Cascadilla Proceedings Project, 100-109.

Cheng, A. C., Lu, H.-C. and Giannakouros, P. (2008). "The uses of Spanish copulas by Chinesespeaking learners in a free writing task", in Bilingualism: Language and Cognition, 11(3): 301-317.

Finneman, M. (1990). "Markedness and learner strategy: Form and meaning-oriented learners in the foreign language context", in Modern Language Journal, 74: 176-187.

Geeslin, K. (2000). "A new approach to the second language acquisition of copula choice in Spanish", in R. Leow and C. Sanz (eds.), Spanish applied linguistics at the turn of the millennium: Papers from the 1999 conference on the L1 \& L2 acquisition of Spanish and Portuguese. Somerville: Cascadilla Press, 50-66.

Geeslin, K. (2002a). "Semantic transparency as a predictor of copula choice in second language acquisition", in Linguistics: An Interdisciplinary Journal of the Language Sciences, 40(2): 439-468.

Geeslin, K. (2002b). "The second language acquisition of copula choice and its relationship to language change", in Studies in Second Language Acquisition, 24(3): 419-451.

Geeslin, K. (2003a). "A comparison of copula choice in advanced and native Spanish", in Language Learning, 53(4): 703-764.

Geeslin, K. (2003b). "The role of adjectival features in the second language acquisition of copula choice", in P. Kempchinsky and C. Piñeros, (eds.), Theory, practice and acquisition: Papers from the 6th Hispanic linguistics symposium and the 5th conference on the acquisition of Spanish and Portuguese. Somerville: Cascadilla Press, 332-351.

Geeslin, K. (2004). Crossing disciplinary boundaries to improve the analysis of second language data: A study of copula choice with adjectives in Spanish. Munich: LINCOM Europa Publisher.

Geeslin, K. and Guijarro-Fuentes, P. (2004). "Estudio Longitudinal del ser y estar en el Español como L2", in Porta Linguarum: Revista Internacional de Didáctica de las Lenguas Extranjeras, 2: 93-110.

Geeslin, K. and Guijarro-Fuentes, P. (2005). "The acquisition of copula choice in instructed Spanish: The role of individual characteristics", in D., Eddington (ed.), Studies in the acquisition of the Hispanic languages: Papers from the 6th conference on the acquisition of Spanish and Portuguese as first and second languages. Somerville: Cascadilla Press, 66-77.

Geeslin, K. and Guijarro-Fuentes, P. (2006). "The second language acquisition of variable structures in Spanish by Portuguese speakers", in Language Learning, 56(1): 53-107.

Gunterman, G. (1992). "An analysis of interlanguage development over time: Part II, ser and estar", in Hispania, 75: 1294-1303.

Odlin, T. (1989). Language tranfer: Cross-linguistic influence in language learning._Cambridge: Cambridge University Press.

Ramírez-Gelpi, A. (1995). The acquisition of ser and estar among adult native English speakers learning Spanish as a second language. (Unpublished Ph.D. Dissertation). University of Southern California: Los Angeles.

Ringbom, H. (1986). "Crosslinguistic influence and the foreign language learning process", in E. Kellerman, and M. Sharwood-Smith (eds.), Crosslinguistic influence in second language acquisition. Oxford: Pergamon Press, 150-162.

Ringbom, H. (1987). The role of the first language in foreign language learning. Clevedon: Multilingual Matters.

Ringbom, H. (2007). Cross-linguistics similarity in foreign language learning. Clevedon: Multilingual Matters. 
Ryan, J. and Lafford, B. (1992). "The acquisition of lexical meaning in a study abroad environment: Ser + estar and the Granada experience", Hispania, 75: 714-22.

Solé, Y. R., and Solé, C. A. (1977). Modern Spanish syntax: A study in contrast. Lexington, Mass: Heath

VanPatten, B. (1985). "The acquisition of "ser" and "estar" by adult learners of Spanish: A preliminary investigation of transitional stages of competence", Hispania, 68(2): 399-406.

VanPatten, B. (1987). "Classroom learners' acquisition of ser and estar: Accounting for developmental patterns", in B. VanPatten, T. R. Dvorak and J. F. Lee (eds.), Foreign language learning: A research perspective. Rowley: Newbury House, 61-75.

William S. and Hammarberg, B. (1998). "Language switches in L3 production: implications for a polyglot speaking model", Applied Linguistics, 19: 295-333.

Woolsey, D. (2006a). Controlling contexts and clarifying intent: Instrument design and the study of the SLA of estar with adjectives. Selected proceedings of the 7th conference on the acquisition of Spanish and Portuguese as first and second languages. Somerville: Cascadilla Press.

Woolsey, D. (2006b). Development of learner use of 'estar + adjective' in contexts of comparison within an individual frame of reference. Selected proceedings of the 9th Hispanic linguistics symposium. Somerville: Cascadilla Press.

CEATE. http://corpora.flld.ncku.edu.tw/

Corpus Tool de UAM. http://www.wagsoft.com/CorpusTool/ 


\section{Apéndice 1}

Número:

Composición A: (22 minutos)
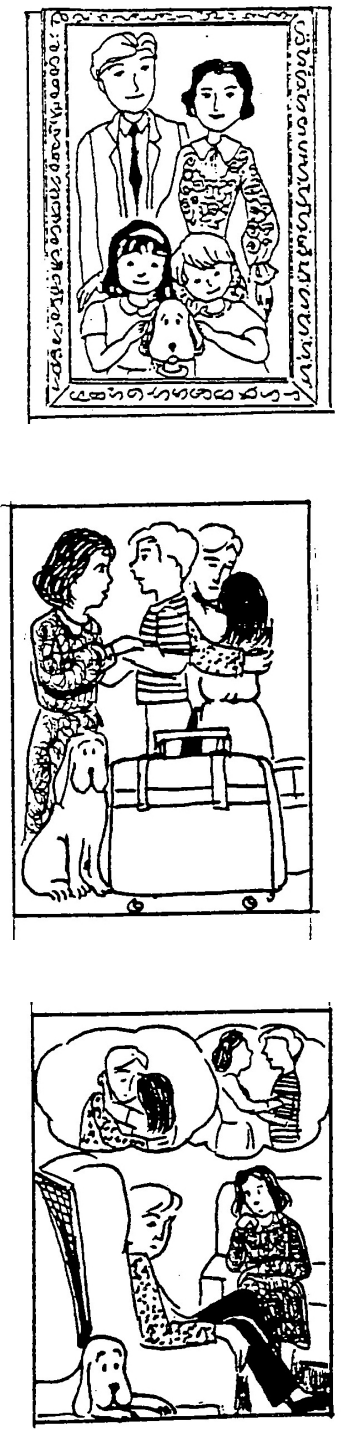

1. la familia: tradicional

2. hijos (Carlos y María): obedientes (順從的)
3. Carlos y María: mayores

4. hijos: ir a la universidad

5. padres: tristes
6. padres: recordar (想起), hijos

7. imágenes: similares

8. perro: solo (孤單的)

9. la vida: demasiado tranquila

10. todos: nostálgicos (想家的) 


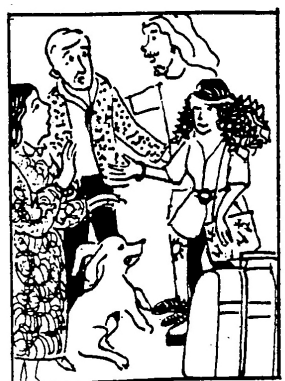

Composición B: (22 minutos)
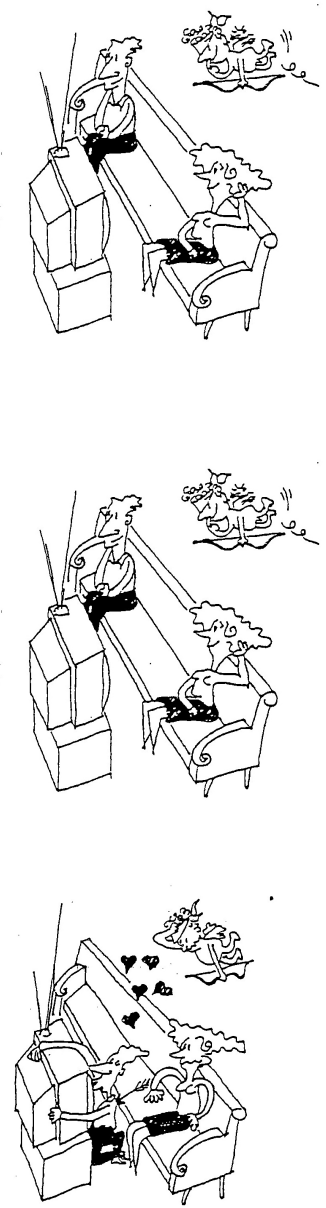

11. padres: sorprendidos (驚訝的)

12. hijos: cambiados

13. la ropa de los hijos: extravagante (離奇古怪

14. el pelo de María: rizado (捲的)

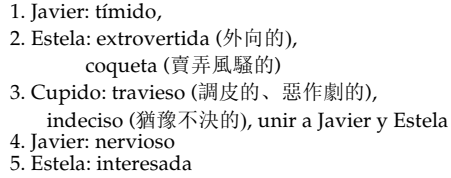

6. Javier: mira su programa, favorito de televisión

7. El programa: entretenido (有趣的)

8. Cupido: lanzar (射)/ flecha (箭)

9. Estela: ilusionada (抱有幻想的、充滿幻想的)

10. El resultado: sorprendente (驚訝的)

11. Javier: enamorado (戀愛著的、愛上某人的)

12. Estela: disgustada (失意的、挫敗的) 